\title{
Gleich viele Fälle von Kindsmisshandlung
}

\section{Markus Wopmann}

Dr. med., Leiter Fachruppe Kinderschutz der Schweizerischen Kinderkliniken, Chefarzt Klinik für Kinder und Jugendliche Kantonsspital Baden

Im Jahre 2015 wurden im siebten Jahr in Folge die Kinder erfasst, die wegen vermuteter oder sicherer Kindsmisshandlung ambulant oder stationär an einer schweizerischen Kinderklinik behandelt worden waren. In diesem Jahr konnten die Daten von 20 von insgesamt 26 Kinderkliniken der Schweiz bearbeitet werden, was einem Rücklauf von $77 \%$ aller Kliniken entspricht. Wiederum haben alle grossen und mittelgrossen Kinderkliniken ihre Fälle gemeldet, so dass die von uns erfassten Fälle einen sehr grossen Teil der Fälle von Kindsmisshandlung darstellen, die im letzten Jahr an schweizerischen Kinderkliniken behandelt worden waren.

\section{Resultate}

Von den 20 Kliniken wurden insgesamt 1388 Fälle gemeldet. Die Zahlen einer Klinik, die letztes Jahr 23 Fälle gemeldet hatte, standen dieses Jahr nicht zur Verfügung, so dass die Anzahl der Fälle sicher der Zahl des letzten Jahres entspricht. Die Zahlen aller meldenden Kliniken sind gegenüber dem Vorjahr etwa gleich geblieben, so dass möglicherweise ein gewisses Plateau (auf leider hohem Niveau!) erreicht worden ist. In

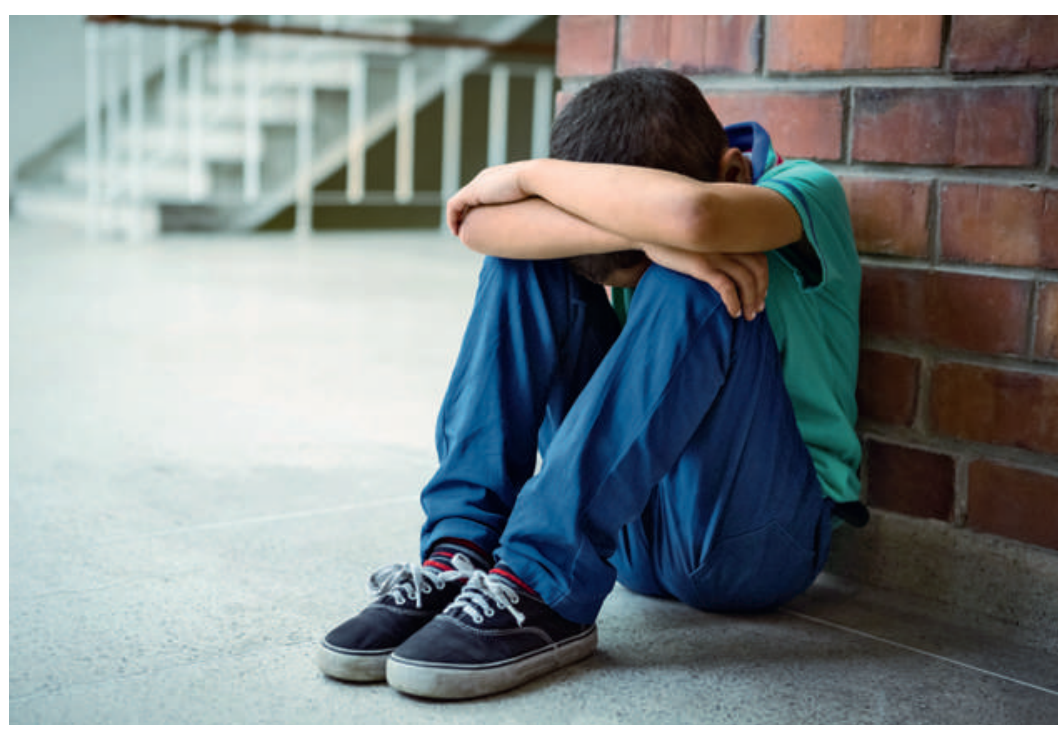

Der Anteil psychisch misshandelter Kinder hat zugenommen. den verschiedenen Untergruppen ergaben sich folgende Zahlen:

\begin{tabular}{lrr}
\hline Körperliche Misshandlung & 393 & $(28,3 \%)$ \\
\hline Vernachlässigung & 277 & $(20,0 \%)$ \\
\hline Psychische Misshandlung & 432 & $(31,1 \%)$ \\
\hline Sexueller Missbrauch & 276 & $(19,9 \%)$ \\
\hline Münchhausen-Stellvertreter-Syndrom & 10 & $(0,7 \%)$
\end{tabular}

Wiederum hat der Anteil der psychisch misshandelten Kinder zugenommen, so dass diese Misshandlungsform nun den grössten Anteil aller Misshandlungen ausmacht. Dies ist wahrscheinlich darauf zurückzuführen, dass zunehmend mehr Kinderkliniken auch in die Abklärungen von Fällen häuslicher Gewalt eingebunden sind, wo ja viele Kinder durch das Miterleben von Gewalt zwischen den Elternteilen zumindest psychisch stark betroffen sind. Fast $1 / 4$ aller misshandelten Kinder war jünger als zwei Jahre alt, $45 \%$ der misshandelten Kinder sind jünger als sechs Jahre. Mit 43\% Knaben und 57\% Mädchen ist die Geschlechterverteilung sehr ähnlich wie in den letzten Jahren.

\begin{tabular}{lrr}
\hline \multicolumn{3}{l}{ Geschlecht der Kinder in den einzelnen Diagnosegruppen } \\
\hline Körperliche Misshandlung & Knaben & Mädchen \\
\hline Vernachlässigung & $56,7 \%$ & $43,3 \%$ \\
\hline Psychische Misshandlung* & $44,4 \%$ & $55,6 \%$ \\
\hline Sexueller Missbrauch & $46,3 \%$ & $53,2 \%$ \\
\hline
\end{tabular}

* In 0,5\% der Fälle wurde das Geschlecht des Kindes nicht erfasst.

Wiederum ist der Anteil der geschlagenen Knaben höher als der der Mädchen, bei den übrigen Misshandlungsformen ist der Mädchenanteil höher, insbesondere beim sexuellen Missbrauch, wo dieses Jahr fast fünfmal mehr Mädchen als Knaben betroffen waren.

Sicherheit der Diagnose*

\begin{tabular}{lll}
\hline Sicher & 842 & $(60,7 \%)$ \\
\hline Wahrscheinlich & 298 & $(21,5 \%)$ \\
\hline Unklar & 248 & $(17,2 \%)$
\end{tabular}

* In 0,6\% der Fälle wurde keine Angabe zur Sicherheit der Diagnose gemacht. 
Die Diagnose psychische Misshandlung wurde mit 75\% am ehesten als sicher eingestuft, körperliche Misshandlung galt als sicher in 59\% der Fälle, Vernachlässigung in $61 \%$ der Fälle. Beim sexuellen Missbrauch war die Sicherheit der Diagnose nur in $42 \%$ der Fälle gegeben.

\begin{tabular}{lrr}
\hline Täterin/Täter: Beziehung zum Kind & & \\
\hline Familie & 1016 & $(80,3 \%)$ \\
\hline Bekannte/Bekannter des Kindes & 166 & $(12,0 \%)$ \\
\hline Fremdtäter & 33 & $(2,4 \%)$ \\
\hline Unbekannter Täter & 73 & $(5,3 \%)$
\end{tabular}

Weiterhin finden psychische Misshandlung und Vernachlässigung in praktisch allen Fällen im Familienrahmen statt, die körperliche Misshandlung in $3 / 4$ der Fälle, sexueller Missbrauch in $46 \%$ der Fälle. 20\% der sexuellen Übergriffe werden durch Fremdtäter oder unbekannte Täter begangen.

\begin{tabular}{lll}
\hline Täterin/Täter: Geschlecht & & \\
\hline Männlich & 639 & $(46,0 \%)$ \\
\hline Weiblich & 350 & $(25,2 \%)$ \\
\hline $\begin{array}{l}\text { Männlich und weiblich } \\
\text { (meist Eltern gemeinsam) }\end{array}$ & 281 & $(20,2 \%)$ \\
\hline Unbekannt & 180 & $(8,6 \%)$
\end{tabular}

Der Anteil der männlichen Täter beim sexuellen Missbrauch lag mit knapp 85\% in dem Rahmen wie in anderen nationalen oder internationalen Studien. Bei der Vernachlässigung werden in $45 \%$ die Mütter und in $39 \%$ beide Elternteile zusammen verantwortlich ge-

\section{$20 \%$ der sexuellen Übergriffe werden durch Fremdtäter oder unbekannte Täter begangen.}

macht, bei der psychischen Misshandlung und bei der körperlichen Misshandlung ist der Anteil der Männer als Täter mit rund 45\% höher als der Anteil der Frauen. Für die insgesamt zehn Fälle von Münchhausen-Stellvertreter-Syndrom wurden schliesslich Frauen als Täterinnen erfasst, was der internationalen Literatur entspricht.

Korrespondenz: Dr. med. Markus Wopmann Leiter der Fachgruppe Chefarzt der Klinik für Kinder und Jugendliche Kantonsspital CH-5404 Baden markus.wopmann[at]ksb.ch

\begin{tabular}{lrr}
\hline Täterin/Täter: Alter & & \\
\hline Älter als 18 Jahre & ca. 1157 & (ca. 83\%) \\
\hline Jünger als 18 Jahre & ca. 130 & (ca. 9\%) \\
$\begin{array}{l}\text { Jünger und älter als 18 Jahre } \\
\text { (mehrere Täter) }\end{array}$ & 8 & $(0,6 \%)$ \\
\hline \begin{tabular}{l} 
Unbekanntes Alter / keine Angabe \\
\hline
\end{tabular}
\end{tabular}

Etwa gleichbleibender Anteil der jugendlichen Täter, wobei diese - wie in den letzten Jahren - beim sexuellen Missbrauch mit über 20\% deutlich mehr vertreten sind als in den anderen Misshandlungsformen.

\begin{tabular}{|c|c|c|}
\hline Vormundschaftliche Massnahmen & & \\
\hline $\begin{array}{l}\text { Durch eine andere Stelle } \\
\text { bereits eingeleitet }\end{array}$ & 338 & $(24,4 \%)$ \\
\hline $\begin{array}{l}\text { Gefährdungsmeldung durch die } \\
\text { Kinderschutzgruppe gemacht }\end{array}$ & 338 & $(24,4 \%)$ \\
\hline $\begin{array}{l}\text { Gefährdungsmeldung durch die } \\
\text { Kinderschutzgruppe empfohlen }\end{array}$ & 91 & $(6,6 \%)$ \\
\hline \multicolumn{3}{|l|}{ Strafrechtliche Massnahmen } \\
\hline $\begin{array}{l}\text { Durch andere Stellen bereits } \\
\text { eingeleitet }\end{array}$ & 286 & $(20,6 \%)$ \\
\hline $\begin{array}{l}\text { Durch die Kinderschutzgruppe } \\
\text { veranlasst }\end{array}$ & 85 & $(6,1 \%)$ \\
\hline $\begin{array}{l}\text { Durch die Kinderschutzgruppe } \\
\text { empfohlen }\end{array}$ & 29 & $(2,1 \%)$ \\
\hline
\end{tabular}

\section{Fazit}

- Die Anzahl der an schweizerischen Kinderkliniken behandelten Kinder mit Kindsmisshandlung blieb auf unverändert hohem Niveau stabil.

- Mit über 30\% der Fälle ist die psychische Misshandlung die häufigste an Kinderkliniken erfasste Misshandlungsform geworden. Dies ist mit hoher Wahrscheinlichkeit im Zusammenhang mit den sehr vielen Fällen von häuslicher Gewalt zu sehen, wo Kinder mindestens indirekt mitbetroffen sind.

- In jedem 4. Fall hat die Kinderschutzgruppe selber eine Gefährdungsmeldung an die Kindes- und Erwachsenenschutzbehörde gemacht, genau so häufig war diese Behörde auch vorher schon eingeschaltet. Dies passt zu der Tatsache, dass die Kindsmisshandlung - abgesehen vom sexuellen Missbrauch - häufig in sozial benachteiligten Familien vorkommt.

- Im Jahr 2015 ist ein Kind an einer schweizerischen Kinderklinik aufgrund körperlicher Misshandlung verstorben, das Kind war jünger als ein Jahr. Bei 250 weiteren Kindern unter einem Jahr (entspricht 18,1\% aller betroffenen Kinder!) wurde die Diagnose Kindsmisshandlung gestellt, womit der Anteil der sehr kleinen Kinder wiederum erschreckend hoch ist.

\section{Bildnachweis}

(c) Wavebreakmediamicro | Dreamstime.com 